\title{
Design of 2D-Bootlace Lens with Five Focal Feed for Multiple Beam Forming
}

\author{
Ravi Pratap Singh Kushwah, P. K. Singhal
}

Microwave Laboratory, Department of Electronics, Madhav Institute of Technology \& Science, Gwalior, India.

Email: kushwah.ravipratapsingh@rediffmail.com

Received January $10^{\text {th }}, 2011$; revised January $20^{\text {th }}, 2011$; accepted January $25^{\text {th }}, 2011$

\begin{abstract}
Utility of microwave bootlace lens is well established. For broadband and wide scanning network, it is required to optimize and use features of this lens for better communication and scanning device. This report presents the design of a compact $2 D$ bootlace lens with five focal feed for multiple beam forming. This design will help in reducing phase error and will provide power efficiency and better resolution during target tracking. This design will provide better aperture efficiency and scanning angle.
\end{abstract}

Keywords: Antenna Array, Rotman Lens, Multiple Beam Forming, Wide Angle Scanning

\section{Introduction}

In many radar and communication applications, it is often required to scan a wide area using an antenna array. A multiple beam-forming network is required to control the amplitude and phase at each element of the antenna array. Microwave bootlace lens forms an important class of multiple beam forming networks. Ruze [1] suggested a lens for wide-angle scanning. Rotman and Turner [2,3] suggested modification in Ruze's lens to improve the scanning capabilities. Wide-angle scanning capabilities of these lenses are well established [4-6]. P. K. Singhal et al. [7] suggested modified design equations to improve the performance of the bootlace lens.

Bootlace lenses have traditionally offered great design flexibility without the excessive cost and reliability difficulties of phased array. This present report proposes to improve the current lens technology by developing a five focal point lens with scanning capability in a plane. As with previously developed multifocal bootlace lenses, specifying several adjacent perfectly focused scanned beam gives a well-focused field of view across the region bounded by the focal points. The entire aperture is used for all beams, providing optimum efficiency for this antenna.

\section{Lens Design}

Figure 1 shows the geometry of five focal point bootlace lenses. For a lens with five perfect focal points, the focal points are positioned as two pairs in different scan planes

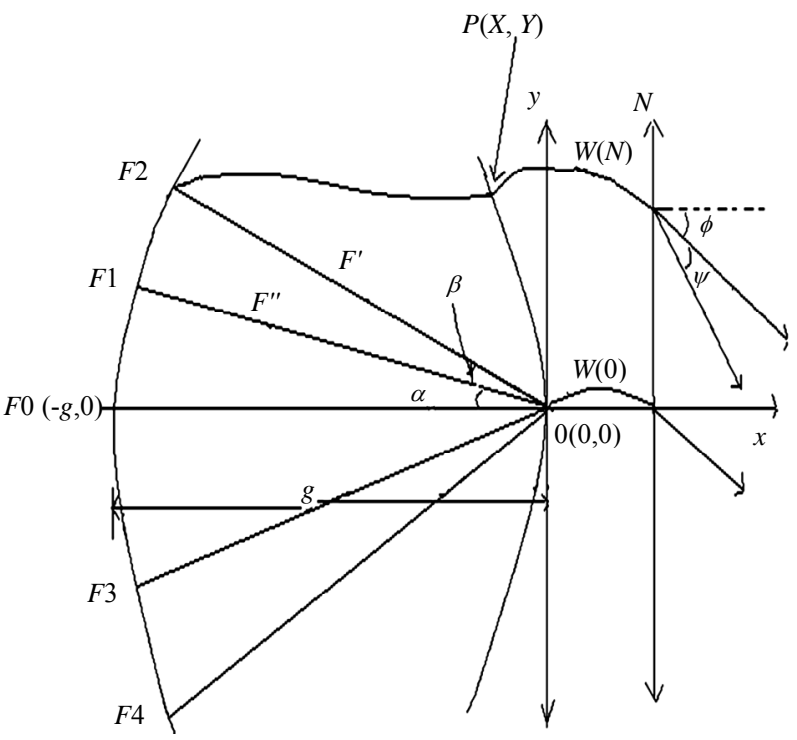

Figure 1. Five feed bootlace lens.

and one central point. Un-scanned focus to accommodate a wide, two-dimensional field of view [8].

Using the design approach suggested in [9] and modified for this design following equations are written:

$$
\begin{aligned}
& \sqrt{\varepsilon_{r}}\left(F_{1} P\right)+\sqrt{\varepsilon_{r e}} w(N)+N \sin \Psi=\sqrt{\varepsilon_{r}} F^{\prime \prime}+\sqrt{\varepsilon_{r e}} w(0)(1) \\
& \sqrt{\varepsilon_{r}}\left(F_{2} P\right)+\sqrt{\varepsilon_{r e}} w(N)+N \sin \Phi=\sqrt{\varepsilon_{r}} F^{\prime}+\sqrt{\varepsilon_{r e}} w(0)(2) \\
& \sqrt{\varepsilon_{r}}\left(F_{3} P\right)+\sqrt{\varepsilon_{r e}} w(N)-N \sin \Psi=\sqrt{\varepsilon_{r}} F^{\prime \prime}+\sqrt{\varepsilon_{r e}} w(0)(3)
\end{aligned}
$$




$$
\begin{aligned}
& \sqrt{\varepsilon_{r}}\left(F_{4} P\right)+\sqrt{\varepsilon_{r e}} w(N)-N \sin \Phi=\sqrt{\varepsilon_{r}} F^{\prime}+\sqrt{\varepsilon_{r e}} w(0)(4) \\
& \sqrt{\varepsilon_{r}}\left(F_{0} P\right)+\sqrt{\varepsilon_{r e}} w(N)=\sqrt{\varepsilon_{r}} G+\sqrt{\varepsilon_{r e}} w(0)
\end{aligned}
$$

where

$$
\begin{aligned}
& \left(F_{1} P\right)^{2}=\left(X+F^{\prime \prime} \cos \alpha\right)^{2}+\left(Y-F^{\prime \prime} \sin \alpha\right)^{2} \\
& \left(F_{2} P\right)^{2}=\left(X+F^{\prime} \cos \beta\right)^{2}+\left(Y-F^{\prime} \sin \beta\right)^{2} \\
& \left(F_{3} P\right)^{2}=\left(X+F^{\prime \prime} \cos \alpha\right)^{2}+\left(Y+F^{\prime \prime} \sin \alpha\right)^{2} \\
& \left(F_{2} P\right)^{2}=\left(X+F^{\prime} \cos \beta\right)^{2}+\left(Y+F^{\prime} \sin \beta\right)^{2} \\
& \left(F_{0} P\right)^{2}=(X+G)^{2}+(Y)^{2}
\end{aligned}
$$

The five path length equation (counting each choice of ' \pm ' as a single equation) are now simplified and solved numerically to give the surface and transmissions line length

$F=$ Distance from $F_{1}$ to $O \& F_{2}$ to $O$ called the off-axis focal length.

$G=$ Distance from $F_{0}$ to $O$ called the on-axis focal length.

$N=$ Indicate the position of the radiating elements, called the lens aperture.

$\varepsilon_{r}=$ Substrate dielectric constant.

$\varepsilon_{r e}=$ Effective dielectric constant of the transmission lines.

Design parameters are normalized relative to the maximum lens aperture $N_{\max }$ and defined as follows:

$$
\begin{aligned}
& f=F / N_{\max } \\
& g=G / N_{\max } \\
& w=(W(0)-W(N)) \sqrt{\varepsilon_{r e}} /\left(\sqrt{\varepsilon_{r}} \cdot N_{\max }\right) \\
& \eta=N / N_{\max } \\
& y=Y / N_{\max } \text { and } \\
& x=X / N_{\max } \\
& f^{\prime}=F_{2} / N_{\max } \\
& f^{\prime \prime}=F_{1} / N_{\max }
\end{aligned}
$$

Algebraic manipulation of the equations above gives

$$
\begin{aligned}
\left(F_{0} P\right)^{2}= & (X+G)^{2}+(G)^{2}+2 X G+(Y)^{2} \\
\left(F_{1} P\right)^{2}= & (X)^{2}+\left(F^{\prime \prime} \cos \alpha\right)^{2}+2 X F^{\prime \prime} \cos \alpha+(Y)^{2} \\
& +\left(F^{\prime \prime} \sin \alpha\right)^{2}-2 F^{\prime \prime} \sin \alpha \\
\left(F_{2} P\right)^{2}= & (X)^{2}+\left(F^{\prime} \cos \beta\right)^{2}+2 X F^{\prime} \cos \beta+(Y)^{2} \\
& +\left(F^{\prime} \sin \beta\right)^{2}-2 F^{\prime} \sin \beta
\end{aligned}
$$

$$
\begin{aligned}
\left(F_{3} P\right)^{2}= & (X)^{2}+\left(F^{\prime \prime} \cos \alpha\right)^{2}+2 X F^{\prime \prime} \cos \alpha+(Y)^{2} \\
& +\left(F^{\prime \prime} \sin \alpha\right)^{2}+2 F^{\prime \prime} \sin \alpha \\
\left(F_{4} P\right)^{2}= & (X)^{2}+\left(F^{\prime} \cos \beta\right)^{2}+2 X F^{\prime} \cos \beta+(Y)^{2} \\
& +\left(F^{\prime} \sin \beta\right)^{2}+2 F^{\prime} \sin \beta
\end{aligned}
$$

Normalized by $N_{\max }$

$$
\begin{aligned}
& \left(F_{1} P\right)^{2}=\left(X+F^{\prime \prime} \cos \alpha\right)^{2}+\left(Y-F^{\prime \prime} \sin \alpha\right)^{2} \\
& \left(F_{2} P\right)^{2}=\left(X+F^{\prime} \cos \beta\right)^{2}+\left(Y-F^{\prime} \sin \beta\right)^{2} \\
& \left(F_{3} P\right)^{2}=\left(X+F^{\prime \prime} \cos \alpha\right)^{2}+\left(Y+F^{\prime \prime} \sin \alpha\right)^{2} \\
& \left(F_{4} P\right)^{2}=\left(X+F^{\prime} \cos \beta\right)^{2}+\left(Y+F^{\prime} \sin \beta\right)^{2} \\
& \left(F_{0} P\right)^{2}=(X+G)^{2}+(Y)^{2}
\end{aligned}
$$

Now by the Equations (1-5)

$$
\begin{aligned}
& \left(F_{1} P / N_{\max }\right)^{2}=w-\eta \sin \psi+f^{\prime \prime} \\
& \left(F_{2} P / N_{\max }\right)^{2}=w-\eta \sin \Phi+f^{\prime} \\
& \left(F_{3} P / N_{\max }\right)^{2}=w+\eta \sin \psi+f^{\prime \prime} \\
& \left(F_{4} P / N_{\max }\right)^{2}=w+\eta \sin \Phi+f^{\prime} \\
& \left(F_{0} P / N_{\max }\right)^{2}=w+g
\end{aligned}
$$

Squaring (21) to (25)

$$
\begin{aligned}
& \left(F_{1} P / N_{\text {max }}\right)^{2}=(w)^{2}+\left(f^{\prime \prime}\right)^{2}+(\eta \sin \psi)^{2}-2 \eta f^{\prime \prime} \sin \psi \\
& -2 w \eta \sin \psi+2 w f^{\prime \prime} \\
& \left(F_{2} P / N_{\text {max }}\right)^{2}=(w)^{2}+\left(f^{\prime}\right)^{2}+(\eta \sin \Phi)^{2}-2 \eta f^{\prime} \sin \Phi \\
& -2 w \eta \sin \Phi+2 w f^{\prime} \\
& \left(F_{3} P / N_{\text {max }}\right)^{2}=(w)^{2}+\left(f^{\prime \prime}\right)^{2}+(\eta \sin \psi)^{2}+2 \eta f^{\prime \prime} \sin \psi \\
& \quad+2 w \eta \sin \psi+2 w f^{\prime \prime} \\
& \left(F_{4} P / N_{\text {max }}\right)^{2}=(w)^{2}+\left(f^{\prime}\right)^{2}+(\eta \sin \Phi)^{2}+2 \eta f^{\prime} \sin \Phi \\
& \quad+2 w \eta \sin \Phi+2 w f^{\prime} \\
& \left(F_{0} P / N_{\text {max }}\right)^{2}=(w)^{2}+(g)^{2}+2 w g
\end{aligned}
$$

Equating both $\left(F_{0} P / N_{\max }\right)^{2}$ by Equation (25), (30)

$$
(x)^{2}-(w)^{2}+(y)^{2}=2 g(w-x)
$$

Equating both (16) \& (26), (17) \& (27), (18) \& (28), 
(19) \& (29) and putting the value of $(x)^{2}-(w)^{2}+(y)^{2}$ by Equation (31)

$$
\begin{aligned}
& 2 x f^{\prime \prime} \cos \alpha-2 y f^{\prime \prime} \sin \alpha-2 x g+2 w g \\
& =(\eta \sin \psi)^{2}-2 \eta f^{\prime \prime} \sin \psi-2 w \eta \sin \psi+2 w f^{\prime \prime} \\
& 2 x f^{\prime} \cos \beta-2 y f^{\prime} \sin \beta-2 x g+2 w g \\
& =(\eta \sin \Phi)^{2}-2 \eta f^{\prime \prime} \sin \Phi-2 w \eta \sin \Phi+2 w f^{\prime} \\
& 2 x f^{\prime \prime} \cos \alpha+2 y f^{\prime \prime} \sin \alpha-2 x g+2 w g \\
& =(\eta \sin \psi)^{2}+2 \eta f^{\prime \prime} \sin \psi+2 w \eta \sin \psi+2 w f^{\prime \prime} \\
& 2 x f^{\prime} \cos \beta+2 y f^{\prime} \sin \beta-2 x g+2 w g \\
& =(\eta \sin \Phi)^{2}+2 \eta f^{\prime \prime} \sin \Phi+2 w \eta \sin \Phi+2 w f^{\prime} \\
& \text { Add (32) \& (34), (33) \& (35) } \\
& 2 x f^{\prime \prime} \cos \alpha-2 x g+2 w g=(\eta \sin \psi)^{2}+2 w f^{\prime \prime} \\
& 2 x f^{\prime} \cos \beta-2 x g+2 w g=(\eta \sin \Phi)^{2}+2 w f^{\prime} \\
& y f^{\prime \prime} \sin \alpha=\eta f^{\prime \prime} \sin \psi+w \eta \sin \psi \\
& y f^{\prime} \sin \beta=\eta f^{\prime} \sin \Phi+w \eta \sin \Phi
\end{aligned}
$$

By (38) \& (39)

$$
\begin{aligned}
& y=\eta \sin \psi\left(w+f^{\prime \prime}\right) / f^{\prime \prime} \sin \alpha ; \\
& \eta \sin \Phi\left(w+f^{\prime}\right) / f^{\prime} \sin \beta
\end{aligned}
$$

By (36) \& (37)

$$
\begin{aligned}
& x=(\eta \sin \psi)^{2}+2 w\left(f^{\prime \prime}-g\right) / 2\left(f^{\prime \prime} \cos \alpha-g\right) ; \\
& (\eta \sin \Phi)^{2}+2 w\left(f^{\prime}-g\right) / 2\left(f^{\prime} \cos \beta-g\right)
\end{aligned}
$$

Value of $x \& y$ put into Equation (31) and solve then we get the equation

$$
\begin{aligned}
& {\left[\left\{\left(f^{\prime}-g\right)^{2} /\left(f^{\prime} \cos \beta-g\right)^{2}\right\}\right.} \\
& \left.+\left\{\left(\eta / f^{\prime}\right)^{2}(\sin \Phi / \sin \beta)^{2}\right\}-1\right] w^{2} \\
& +\left[\left\{(\eta \sin \Phi)^{2}\left(f^{\prime}-g\right) /\left(f^{\prime} \cos \beta-g\right)^{2}\right\}\right. \\
& +\left\{2(\eta \sin \Phi)^{2} / f^{\prime}(\sin \beta)^{2}\right\}+\left\{2 g\left(f^{\prime}-g\right) /\left(f^{\prime} \cos \beta-g\right)\right\} \\
& -2 g] w+\left[\left\{(\eta \sin \Phi)^{4} /\left(f^{\prime} \cos \beta-g\right)^{2}\right\}\right. \\
& \left.+\left\{\left(\eta^{2} / f^{\prime}\right)(\sin \Phi / \sin \beta)^{2}\right\}+\left\{g(\eta \sin \Phi)^{2} /\left(f^{\prime} \cos \beta-g\right)\right\}\right] \\
& =0
\end{aligned}
$$

\section{Compare}

$$
A w^{2}+B w+C=0
$$

where

$$
\begin{aligned}
A= & \left\{\left(f^{\prime}-g\right)^{2} /\left(f^{\prime} \cos \beta-g\right)^{2}\right\} \\
& +\left\{\left(\eta / f^{\prime}\right)^{2}(\sin \Phi / \sin \beta)^{2}\right\}-1 \\
B= & \left\{(\eta \sin \Phi)^{2}\left(f^{\prime}-g\right) /\left(f^{\prime} \cos \beta-g\right)^{2}\right\} \\
& +\left\{2(\eta \sin \Phi)^{2} / f^{\prime}(\sin \beta)^{2}\right\} \\
& +\left\{2 g\left(f^{\prime}-g\right) /\left(f^{\prime} \cos \beta-g\right)\right\}-2 g \\
C= & \left\{(\eta \sin \Phi)^{4} /\left(f^{\prime} \cos \beta-g\right)^{2}\right\} \\
& +\left\{\left(\eta^{2} / f^{\prime}\right)(\sin \Phi / \sin \beta)^{2}\right\} \\
& +\left\{g(\eta \sin \Phi)^{2} /\left(f^{\prime} \cos \beta-g\right)\right\}
\end{aligned}
$$

Again Value of $x \& y$ put into Equation (40) and solve then we get the equation

$$
\begin{aligned}
& {\left[\left\{\left(f^{\prime \prime}-g\right)^{2} /\left(f^{\prime \prime} \cos \alpha-g\right)^{2}\right\}\right.} \\
& \left.+\left\{\left(\eta / f^{\prime \prime}\right)^{2}(\sin \psi / \sin \alpha)^{2}\right\}-1\right] w^{2} \\
& +\left[\left\{(\eta \sin \psi)^{2}\left(f^{\prime \prime}-g\right) /\left(f^{\prime \prime} \cos \alpha-g\right)^{2}\right\}\right. \\
& +\left\{2(\eta \sin \psi)^{2} / f^{\prime \prime}(\sin \alpha)^{2}\right\}+\left\{2 g\left(f^{\prime \prime}-g\right) /\left(f^{\prime \prime} \cos \alpha-g\right)\right\} \\
& -2 g] w+\left[\left\{(\eta \sin \psi)^{4} /\left(f^{\prime \prime} \cos \alpha-g\right)^{2}\right\}\right. \\
& +\left\{\left(\eta^{2} / f^{\prime \prime}\right)(\sin \psi / \sin \alpha)^{2}\right\} \\
& \left.+\left\{g(\eta \sin \psi)^{2} /\left(f^{\prime \prime} \cos \alpha-g\right)\right\}\right]=0
\end{aligned}
$$

Compare

where

$$
A w^{2}+B w+C=0
$$

$$
\begin{aligned}
A= & \left\{\left(f^{\prime \prime}-g\right)^{2} /\left(f^{\prime \prime} \cos \alpha-g\right)^{2}\right\} \\
& +\left\{\left(\eta / f^{\prime \prime}\right)^{2}(\sin \psi / \sin \alpha)^{2}\right\}-1 \\
B= & \left\{(\eta \sin \psi)^{2}\left(f^{\prime \prime}-g\right) /\left(f^{\prime \prime} \cos \alpha-g\right)^{2}\right\} \\
& +\left\{2(\eta \sin \psi)^{2} / f^{\prime \prime}(\sin \alpha)^{2}\right\} \\
& +\left\{2 g\left(f^{\prime \prime}-g\right) /\left(f^{\prime \prime} \cos \alpha-g\right)\right\}-2 g
\end{aligned}
$$




$$
\begin{aligned}
C= & \left\{(\eta \sin \psi)^{4} /\left(f^{\prime \prime} \cos \alpha-g\right)^{2}\right\} \\
& +\left\{\left(\eta^{2} / f^{\prime \prime}\right)(\sin \psi / \sin \alpha)^{2}\right\} \\
& +\left\{g(\eta \sin \psi)^{2} /\left(f^{\prime \prime} \cos \alpha-g\right)\right\}
\end{aligned}
$$

\section{Simulated Result}

Based on design equations the proposed design has been simulated for the following parametric values:

$$
\begin{aligned}
& g=1.795 \\
& f 1=1.705 \\
& f 2=1.75 \\
& \varepsilon_{\mathrm{r}}=3.7 \\
& \text { alpha1 }=35
\end{aligned}
$$

and the $f 3$ and $f 4$ are opposite to the $f 1$ and $f 2$. The results shown below in Figure 2 it is clearly shown that the beam width has been reduced drastically and will provide further power spectral efficiency and better scanning area with less amount of power, thus reducing the effective cost of the equipment used for interfacing antenna array.

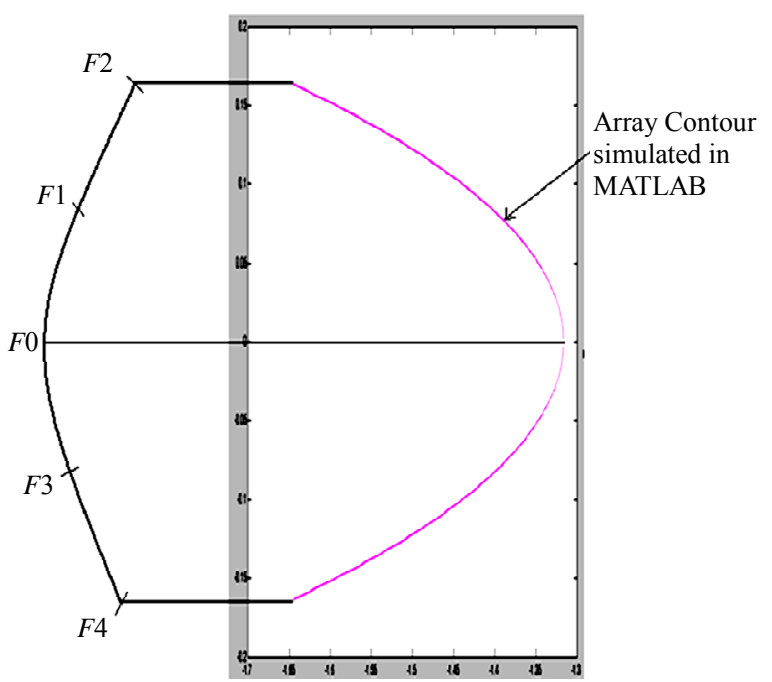

Figure 2. Simulated result of five feed bootlace lens.

\section{Conclusion}

Design equation of 2D-Bootlace lens with five focal feed for multiple beam forming has been computed and pro- posed geometry has been simulated using MATLAB, which gives result as per prediction of this design and will yield wave-front as per specification.

\section{Acknowledgements}

Authors would like to acknowledge the financial support of Dept. of Science and Technology, Govt. of India and the authorities of Madhav Institute of Technology \& Science, Gwalior, India.

\section{REFERENCES}

[1] J. Ruze, "Wide Angle Metal Plate Optics," Proceedings of IRE, Vol. 38, January 1950, pp. 53-59.

[2] W. Rotman and R. F. Turner, "Wide Angle Microwave Lens for Line Source Applications," IEEE Transactions on Antennas Propagation, Vol. AP-11, November 1963, pp. 23-632.

[3] G. Larry Leonakis, "Correction to Wide Angle Microwave Lens for Line Source Applications," IEEE Transactions on Antennas Propagation, Vol. 36, August 1986, p. 1067. doi:10.1109/TAP.1986.1143925

[4] D. Archer, "Lens Fed Multiple Beam Arrays," Microwave Journal, Vol. 18, 1975, pp. 37-42.

[5] D. Archer, "Lens-Fed Multiple-Beam Arrays," Microwave Journal, September 1984, pp. 171-195.

[6] R. D. Gupta, P. C. Sharma and P. K. Singhal, "Bootlace Lens Fed Multiple Beam Antenna: A Review," IETE Technical Review, Vol. 13, No. 4-5, July-October 1996, pp. 207-213.

[7] P. K. Singhal, P. C. Sharma and R. D. Gupta, "Rotman Lens with Equal Height of Array and Feed Contours," IEEE Transactions on Antenna Propagation, Vol. 51, No. 8, August 2003, pp. 2048-2056. doi:10.1109/TAP.2003.814742

[8] C. M. Rappaport and Jeffrey Mason, "A Five Focal Point Three Dimensional Bootlace lens with scanning in two Planes," IEEE Transactions on Antenna and Propagation, 1992, pp. 1340-1343.

[9] M. S. Smith, "Design Consideration for Ruze and Rotman Lenses," The Radio Electronic Engineer, Vol. 52, No. 4, April 1982, pp. 181-187. 